\title{
La responsabilidad social empresarial en la imagen de marca e intención de compra de envases para alimentos y bebidas
}

\section{Corporate social responsibility in the brand image and intention to buy packaging for food and beverages}

Denise María Eguez Ruiz

Universidad de Especialidades Espíritu Santo, Ecuador

Mayra Liuviana Vega Chica

Universidad de Especialidades Espíritu Santo, Ecuador

Autor para correspondencia: deguez@uees.edu.ec, mvegach@uees.edu.ec

Fecha de recepción: 05 de Junio de 2017- Fecha de aceptación: 15 de Noviembre de 2017

Resumen: La aparición de consumidores socialmente responsables que ansían productos que reflejen su consciencia social, ética y moral motiva a las organizaciones a llevar a cabo acciones de Responsabilidad Social Empresarial (RSE). Sin embargo, no se ha analizado la influencia de RSE en la imagen de marca de las empresas ecuatorianas. Adicionalmente, la literatura vigente resulta poco concluyente, en cuanto, a si la realización de este tipo de acciones tiene una influencia considerable en la generación de ventas y en la rentabilidad. A través de la presente investigación la autora busca analizar el impacto de las actividades de responsabilidad social de los fabricantes de envases de vidrio en la intención de compra de las empresas de elaboración de alimentos y bebidas en el Ecuador. Mediante encuestas aplicadas a 258 gerentes de compra, se encuentra que existe una relación entre las acciones de RSE y los factores que afectan la decisión de compra.

Palabras clave: responsabilidad social empresarial (RSE); imagen de marca; rentabilidad; intención de compra

\begin{abstract}
The emergence of socially responsible consumers who crave products that reflect their social, ethical and moral consciousness, encourages organizations to carry out Corporate Social Responsibility (CSR) Actions. However, the influence of CSR on brand image of Ecuadorian companies has not yet been analyzed. In addition, current literature is inconclusive as to whether the realization of such actions has considerable influence in generating sales and profitability. Through this research the author seeks to analyze the impact of social responsibility activities performed by glass containers manufacturers on purchasing intention of food \& beverages manufacturing companies. Through a survey applied to 258 purchasing managers, a relationship between CSR activities and client's evaluation about purchasing factors is found.
\end{abstract}

Key words: corporate social responsibility (CSR); brand image; profitability; purchase intent 


\section{Introducción}

La Responsabilidad Social Empresarial se ha tornado un tema de alto interés para los investigadores del campo de la administración. Desde los años ochenta se ha venido desarrollando el análisis de la influencia de las prácticas de RSE en el comportamiento del consumidor como una línea de investigación en el marketing, dado su impacto en la imagen y reputación de las empresas y sus marcas (Bigne \& Curras, 2008). Sin embargo, poco se ha analizado sobre el impacto de la responsabilidad social corporativa en el caso de empresas en el segmento B2B, es decir, aquellas que proveen productos y/o servicios a otras empresas, y no, al consumidor final. Situación en la cual el impacto de las acciones de RSE de los proveedores podría influir indirectamente en los consumidores finales.

Estos consumidores ansían productos que mejoren o al menos tomen en cuenta la necesidad de proteger a los stakeholders más cercanos para la empresa. De esta manera, el rol de las empresas es observado no solo como una gestión para proveer productos y servicios a un mercado determinado, sino bajo un enfoque integral que busca la generación de bienestar de los diferentes públicos objetivos dentro y fuera de las organizaciones.

Según el ICONTEC (2012), ser socialmente responsable deriva en diversos beneficios tales como: a) Estrechar las relaciones y fidelidad con los stakeholders, b) Promover un modelo dialógico que previene conflictos, c) Propiciar la renovación de la cultura organizacional y d) Proteger y mejorar la reputación de la organización. Adicionalmente, el Parlamento Europeo (2013) inclusive manifiesta que la correcta aplicación de la RSE puede contribuir en gran medida a la recuperación económica sostenible y a mitigar las consecuencias de la crisis económica.

Sin embargo, Correa, Flynt y Amit (2004), encuentran que la RSC continúa siendo un tema de poca importancia para las empresas, sobre todo en regiones como América Latina. De esta manera, la generación de empresas más comprometidas socialmente no se ha dado en igual medida o intensidad en todo el mundo (Méndez, 2005; Vargas, 2006). Cabe destacar que mientras algunos estudios, como IADB (2004) concluyen que la región está atrasada. Otros, en cambio, declaran que los resultados en latinoamérica no difieren en gran medida con los de Estados Unidos o Europa (IFC, 2002).

En Brasil, por ejemplo, una organización sin ánimo de lucro llamada Ethos ha logrado una importante difusión y fomento de la RSE. Esta entidad ha reunido a 1200 pequeñas, medianas y grandes empresas para ayudarles a administrar sus negocios aplicando la RSE. Las mencionadas empresas representan el 35\% del PIB de Brasil, por lo que se han convertido en un elemento clave para la sensibilización sobre esta materia en el resto de países de la región (Aguilar - Platas y Raufflet, 2010). Aun cuando la literatura existente muestra que las acciones sociales influyen más en los países desarrollados que en economías emergentes (Bhattacharya \& Sen, 2004), autores tales como Marquina y Morales (2012) encuentran que la RSE influye positivamente en la compra en contextos latinoamericanos.

La RSE toma particular importancia en sector manufacturero, sobre todo en el sector de alimentos y bebidas, por ser una industria altamente demandante de recursos y por ende ha sido difícil acoplar su estructura a un modelo alineado al desarrollo sostenible (Guardela y Barrios, 2006). Por citar un ejemplo, el sector de Alimentos y Bebidas es el más intensivo en uso de energía, 
lo cual lo convierten en uno de los principales emisores de gases de efecto invernadero (Ministerio de Ambiente, Vivienda y Desarrollo Territorial de Colombia, 2010). Sin embargo, este contexto no limita a que el sector pueda aplicar de forma integral la RSE. Según Fundación Adecco (2014), los ciudadanos de España consideran que las empresas del sector de alimentos y bebidas son las más responsables y las de mejor reputación. De igual manera, López et al. (2011) muestra que las empresas mexicanas del sector alimentario han logrado permanecer más tiempo en el mercado y alcanzaron mejores rendimientos económicos cuando han implementado estrategias de RSE.

Aunque los resultados contradictorios no son concluyentes, la RSE empieza a difundirse en el sector empresarial Ecuatoriano, creando espacios de retribución de la empresa hacia la sociedad, aun cuando los beneficios de su aplicación deben aun ser validados.

La presente investigación busca analizar el impacto de las actividades de responsabilidad social en la imagen de marca y e intención de compra de envases de vidrio bajo la premisa de que la globalización económica y el flujo de información han marcado la aparición de consumidores socialmente responsables (Fernandez \& Merino, 2005). A través de encuestas a una muestra representativa de empresas de elaboración de alimentos y bebidas, se pretende evaluar empíricamente el impacto de la RSE en el Ecuador, concretamente, en el caso de empresas de comercialización de envases de vidrio para alimentos y bebidas. Se aplica el cuestionario diseñado por Bigne et als (2006) aplicado a estudiantes universitarios para medir la influencia de las acciones de RSC en su percepción como consumidores.

La justificación del presente estudio radica en que los progresos que se realicen dentro de esta línea de investigación para profundizar en el impacto de las acciones de RSE en la imagen de marca y en las ventas, apoyarán a incentivar el proceso de adopción de la RSE como parte fundamental de la creación de valor y del pensamiento estratégico.

El presente documento se encuentra dividido en los siguientes apartados: revisión de la literatura, la cual presenta la fundamentación teórica relevante para la investigación, especialmente, en cuanto la evolución de la responsabilidad social y su influencia en las ventas. En la sección de metodología de la investigación se explica el tipo de estudio realizado, así como, las técnicas y herramientas empleadas para la recolección de información. Finalmente, se realiza el informe de resultados donde se plasman los hallazgos, el análisis de la información recabada y el apartado de conclusiones.

\section{Revisión de la literatura}

\section{La Responsabilidad Social Empresarial y su evolución}

Explicar el concepto y las dimensiones de la RSE requiere un proceso analítico complejo debido a la diversidad de perspectivas teóricas existentes (Alvarado, 2007). Inclusive, autores como Kraus y Britzelmaier (2012), manifiestan que no existe un consenso entre las distintas definiciones de RSE. Sin embargo, esto no ha impedido el desarrollo de la responsabilidad social como un campo de investigación en los estudios administrativos (Hadjikhani, Lee y Parks, 2016). Según Cajiga (2011), la RSE es un fenómeno que contribuye de forma activa y voluntaria al mejoramiento social de todas las organizaciones. Según Carroll (2009), Comprende las 
expectativas que la sociedad tiene de las organizaciones en cuatro dimensiones: económicas, legales, éticas y filantrópicas. La dimensión económica, se refiere a las expectativas de rentabilidad de las empresas, mientras la dimensión legal se refiere a la expectativa que tiene la sociedad de que esta rentabilidad se obtenga sujetándose a un marco jurídico. Sin embargo, la sociedad también espera que las actividades empresariales cumplan ciertas normas éticas (dimensión ética) y finalmente, que las empresas voluntariamente lleven a cabo actividades que satisfagan las normas sociales (dimensión filantrópica). Dentro de este contexto, las organizaciones intentan que su accionar real se alinee a las expectativas de sus stakeholders (Cervera, 2007) tratando de influir en su percepción acerca de la realización de actividades de RSE.

La RSE ha sido considerada como una fuente importante de ventaja competitiva por diversos autores en la literatura existente (Bravo et als, 2006; McWilliams et als, 2006; Garriga et als, 2004). Precisamente, por ello el alto interés de los investigadores contemporáneos en cuanto a RSE se debe a su rol en la competencia de mercados (Park et al,2014). La RSE por lo tanto, debería ser implementada y difundida particularmente entornos con mercados fragmentados. Este tipo de mercados contiene productos con ciclos de vida cada vez más cortos y con patrones de consumo cambiantes (Juttner et als, 1994). Las organizaciones no pueden limitarse a simplemente realizar acciones de RSE, sino, que estas deben alinearse a la planificación estratégica de la empresa e inclusive a la gestión de marketing, como actividades generadoras de valor compartido (Porter y Kramer, 2011).

Por otro lado, la RSE es considerada por muchos como una contribución clave del sector empresarial para el desarrollo sostenible (Kolk \& Van Tulder, 2010). Desde 1950, el aumento vertiginoso del impacto del hombre en la naturaleza, las tendencias socio económicas y el aumento de la población (Steffen et al., 2015) han aumentado la preocupación acerca del desarrollo sostenible y por ende también de la RSE.

\section{Imagen de marca y percepción del cliente}

En general, una imagen es un modelo de la realidad que difiere de la realidad misma, ya que, la percepción del sujeto que observa influye en su esquema de realidad (Meyvis et al.,2012). Por lo tanto, la imagen de un objeto no solo depende de su realidad, sino de la percepción de quien la contempla. Por ende, en el entorno empresarial, la imagen de marca debe ser considerada no como aquella imagen que la empresa proyecta, sino como la realidad que el consumidor percibe, aun cuando la realidad y la percepción de la realidad difieran. La imagen de marca es, por lo tanto, el conjunto de percepciones que se expresan como asociaciones en la memoria del consumidor (Keller, 1993). Estas asociaciones pueden ser aplicadas a un producto individual o a una diversidad de ellos (Martinez et al, 2005) Cabe destacar que, en entornos dinámicos que caracterizan al ambiente empresarial vigente, la percepción de los consumidores se ve influenciada por los cambios en los entornos sociales, las estrategias de la empresa y el accionar de los competidores (García, 2002), por lo que, el manejo de la imagen de marca constituye un proceso complejo y dinámico en el cual esta evoluciona constantemente en el tiempo. La imagen de marca, refleja la interacción entre el contexto, los consumidores, los productos y la experiencia previa (Rindell,2013). 
La literatura de marketing manifiesta que las acciones y programas de RSE podrían mejorar la imagen y reputación de la marca ante sus consumidores (Alvarado \& Walesska, 2008). Autores como Polonsky \& Jevons (2006), Lizcano \& Nieto (2006) proponen la existencia de una relación positiva y directa entre la RSE y la imagen de la marca, particularmente en escenarios en los cuales el consumidor es consciente de su entorno y valora su conservación.

De esta manera, los consumidores recompensan las acciones de la empresa (Cervera, 2007), para lo cual, deben conocer y valorar las acciones ejecutadas por la empresa. Por lo tanto, el proceso llevado a cabo dentro de los programas de RSE debe incluir no solo una etapa de ejecución, sino también, un periodo de difusión en el cual se transmite a los diversos públicos objetivos las acciones emprendidas y su impacto. Las organizaciones deben, por ende, ejercer de forma organizada acciones para que la comunidad conozca las actividades de RSE que realiza periódicamente.

Diversas investigaciones muestran que la RSE no solo permite la consecución de una mayor visibilidad e imagen de marca (Ellen, Webb \& Mohr, 2006), sino que también se logra una mejor actitud del consumidor hacia la empresa y sus marcas (Van Herpen, Pennings \& Meulenberg, 2003; Iglesias et al.,2013). De similar manera, la RSE incrementa la identificación entre el consumidor y la empresa (Currás, 2007) y en general disminuye la rigurosidad con la que el consumidor evalúa a la compañía (Mohr \& Webb, 2005).

\section{Responsabilidad Social Empresarial e Intención de compra}

Las acciones y programas de responsabilidad social empresarial (RSE) pueden mejorar la imagen y la reputación de las empresas ante sus consumidores (Alvarado et als, 2008). Sin embargo, aunque la relación entre la RSE y la intención de compra se abordan en la investigación científica, los resultados han sido contradictorios por lo que la literatura no es concluyente (Bigne \& Curras, 2008).

Si bien autores como Fernandez \& Merino (2005) han encontrado una influencia positiva de la RSE en la compra, otros trabajos muestran que no existe una influencia relevante (Carrigan \& Attala, 2001; Bigné et al., 2005). Wright y Ferris (1997) inclusive encontraron una correlación negativa entre la RSE y la rentabilidad financiera de las empresas.

Sin una visión clara de los beneficios estratégicos de la RSE para la rentabilidad de las organizaciones, los directivos permanecerán indispuestos a invertir en RSE para el éxito a largo plazo (Burke \& Logsdon, 1996), por lo que, se vuelve de vital importancia ahondar en el estudio de los beneficios de la RSE, particularmente, en cuanto a su influencia en la generación de ingresos.

\section{Metodología}

El presente artículo aborda el problema de investigación desde un enfoque cuantitativo de tipo transversal y de campo. Se toma como población a las 779 empresas de elaboración de productos alimenticios y bebidas existentes en el Ecuador según la Superintendencia de 
Compañías del Ecuador (2016). Utilizando la fórmula de muestreo aleatorio simple se obtiene un tamaño muestral de 258 encuestas (margen de error: 5\% y nivel de confianza: $95 \%$ ).

En una primera instancia se contactó por vía telefónica a los 258 gerentes de compra, para explicar los objetivos de investigación y confirmar la aceptación de participar en el estudio. También, se consultó a los participantes si preferían realizar la encuesta por vía telefónica, correo electrónico o visita presencial. Finalmente, por requerimiento de los participantes, se envió las encuestas por vía correo electrónico.

Para medir el estado de la aplicación de RSE y la intención de compra en la empresa, se aplica la escala de medición de Bigne et al (2006). El mencionado cuestionario se compone de 5 secciones, tal como lo muestra la tabla 1.

Tabla 1

Secciones del cuestionario

\begin{tabular}{ll}
\hline Secciones & Medición \\
\hline $\begin{array}{l}\text { a. Actividades percibidas como } \\
\text { socialmente responsables }\end{array}$ & $\begin{array}{c}18 \text { items en Escala de } 10 \text { puntos (1: "muy poca } \\
\text { importancia" a 10: "mucha importancia") }\end{array}$ \\
$\begin{array}{l}\text { b. Importancia de diferentes } \\
\text { criterios de compra }\end{array}$ & $\begin{array}{c}10 \text { items en Escala de } 10 \text { puntos (1: "no utilizaré nunca ese } \\
\text { criterio para elegir" a 10: "por supuesto utilizaré ese criterio") }\end{array}$ \\
$\begin{array}{l}\text { c. Nivel y calidad de información } \\
\text { sobre RSC }\end{array}$ & $\begin{array}{c}\text { Una pregunta dicotómica y una pregunta valorando el tipo } \\
\text { de información en escala del 1 al 10 }\end{array}$ \\
$\begin{array}{l}\text { d. Influencia de stakeholders } \\
\text { e. Características } \\
\text { sociodemográficas de los } \\
\text { consumidores }\end{array}$ & $\begin{array}{l}16 \text { items en escala del } 1 \text { al } 10 \\
\text { preguntas cerradas: género, tipo de estudios y nacionalidad }\end{array}$ \\
\hline
\end{tabular}

Fuente: Bigné et als (2006)

Para el presente estudio se adaptan algunos ítems a la industria de envases y se omite las dos últimas secciones, ya que, la encuesta no se realiza a consumidores finales. Al contrario, el estudio de Bigné et als (2006) se realizó aplicando el cuestionario a estudiantes universitarios considerando como productos: zapatos deportivos y desodorantes.

Las actividades socialmente responsables y los criterios de compra que componen los ítems de la herramienta de levantamiento de información, se detallan en las tablas 2 y 3.

Tabla 2.

Actividades socialmente responsables

Actividades/ aplicación de RSC

Invierte en ID en pro del medio ambiente

Reduce despilfarro recursos

Ayuda desarrollo país

Ofrece trato justo

Rechaza actuar países violan DH

Respeta DH 
Contribuye mejora calidad de vida

Cumple todas las leyes

Precisa info sobre composición productos

Crea puestos de trabajo

Tiene código ético de conducta

Ayuda empleados desean formarse

Mejora constantemente calidad

Tratamiento similar a todos los clientes

Publica informe medioambiental anual

Contribuye para actividades socio culturales

Tiene precios bajos

Obtiene los mayores beneficios

Fuente: Bigné et als (2006)

Tabla 3.

Criterios de compra

Criterios de compra

Precio producto

Calidad producto

Calidad servicios

Disponibilidad producto

Implicación en protección medioambiente

Reputación de empresa

Promoción de venta de productos

Contribución empresa a obras sociales

Imagen de Marca

Percepción consumidores sobre producto

Necesidad de disponer de información sobre grado RSC para comprar

Fuente: Bigné et als (2006)

\section{Resultados}

De los gerentes de compra encuestados, el 96,7\% afirmó haber leído sobre responsabilidad social corporativa (el 65,8\% afirmó leer muy frecuentemente).

La tabla número 4 resume la evaluación del criterio de los encuestados acerca de la aplicación de la responsabilidad social corporativa por parte de la empresa que les provee los envases.

Tabla 4.

Aplicación de RSC

\begin{tabular}{ccc}
\hline Ítem & Media & Desviación estándar \\
\hline Invierte en ID en pro del medio ambiente & 8,98 &, 928 \\
Reduce despilfarro recursos & 9,19 &, 875 \\
\hline
\end{tabular}




\begin{tabular}{ccc}
\hline Ayuda desarrollo país & 9,15 &, 990 \\
Ofrece trato justo & 9,26 &, 927 \\
Rechaza actuar países violan DH & 5,60 & 1,535 \\
Respeta DH & 9,33 &, 957 \\
Contribuye mejora calidad de vida & 8,61 &, 988 \\
Cumple todas las leyes & 8,75 &, 967 \\
Precisa info sobre composición productos & 8,71 &, 720 \\
Crea puestos de trabajo & 8,28 &, 914 \\
Tiene código ético de conducta & 9,92 &, 352 \\
Ayuda empleados desean formarse & 9,87 &, 418 \\
Mejora constantemente calidad & 9,80 &, 437 \\
Tratamiento similar a todos los clientes & 7,86 &, 645 \\
Publica informe medioambiental anual & 5,10 &, 639 \\
Tiene precios bajos & 8,27 &, 531 \\
Obtiene los mayores beneficios & 7,37 &, 722 \\
Promedio aplicación RSC & 7,98 &, 320 \\
\hline
\end{tabular}

Fuente: Las encuestas Elaborado por: la autoras

De acuerdo al criterio de los encuestados, se observa que en general sus proveedores mantienen altos niveles de aplicación de actividades de RSC. En una escala del 1 al 10 la empresa obtiene una puntuación de 8,45 en promedio, siendo el puntaje más alto el correspondiente a la tenencia de un código ético de conducta, y el brindar ayuda a los miembros de la empresa para formarse. Al contrario, la puntuación más baja $(5,10)$ denota la falta de publicación de un informe acerca del impacto ambiental. Gráficamente se muestra en el anexo 2 las medias obtenidas en cada ítem.

Por otro lado, al consultar la puntuación que otorgan los encuestados a los diversos criterios de compra considerados en el presente estudio (en base a bigné et als, 2006). Se obtiene que los criterios con mejor valoración son: la calidad y disponibilidad de los productos $(9,67)$, la calidad de servicios $(9,65)$, el precio de los productos $(9,63)$ y la imagen de marca $(9,18)$. Como se muestra en la tabla a continuación:

Tabla 5.

Valoración criterios de compra

\begin{tabular}{ccc}
\hline & Media & Desviación estándar \\
\hline Precio producto & 9,63 &, 626 \\
Calidad producto & 9,67 &, 614 \\
Calidad servicios & 9,65 &, 724 \\
Disponibilidad producto & 9,67 &, 672 \\
Implicación en protección medioambiente & 6,04 &, 726 \\
Reputación de empresa & 7,90 &, 504 \\
Promoción de venta de productos & 6,73 &, 511 \\
Contribución empresa a obras sociales & 6,08 &, 873 \\
Imagen de Marca & 9,18 &, 527 \\
Percepción consumidores sobre producto & 5,48 &, 598 \\
Necesidad de disponer de información sobre grado RSC para comprar & 5,03 &, 348 \\
Promedio criterios de compra & $\mathbf{7 , 7 3 2}$ &, $\mathbf{6 1 1}$ \\
\hline
\end{tabular}

Fuente: Las encuestas

Elaborado por: la autora 
Finalmente, se aplica el estadístico Chi- cuadrado para evaluar si existe una relación entre los items referente a la aplicación de RSC y los items referente a la evaluación de los productos (ver anexo 3). Se encuentra que existe una relación estadísticamente significativa (pvalue<0,05) en los siguientes casos:

Tabla 6.

Prueba Chi-Cuadrado actividades RSC y factores de compra

\begin{tabular}{|c|c|c|}
\hline Actividades de RSC & Factores de compra & pvalue \\
\hline $\begin{array}{l}\text { Invierte en ID en pro del medio } \\
\text { ambiente }\end{array}$ & Implicación en protección medioambiente & 0,004 \\
\hline Ayuda desarrollo país & Reputación de empresa & 0,003 \\
\hline Ayuda desarrollo país & Promoción de venta de productos & 0,008 \\
\hline Ofrece trato justo & Reputación de empresa & 0,009 \\
\hline Rechaza actuar países violan DH & Calidad servicios & 0,026 \\
\hline Contribuye mejora calidad de vida & Implicación en protección medioambiente & 0,015 \\
\hline Contribuye mejora calidad de vida & Imagen de Marca & 0,042 \\
\hline Contribuye mejora calidad de vida & Percepción consumidores sobre producto & 0,046 \\
\hline Cumple todas las leyes & Implicación en protección medioambiente & 0,018 \\
\hline $\begin{array}{l}\text { Precisa info sobre composición } \\
\text { productos }\end{array}$ & Precio producto & 0,017 \\
\hline $\begin{array}{l}\text { Precisa info sobre composición } \\
\text { productos }\end{array}$ & Percepción consumidores sobre producto & 0,018 \\
\hline Crea puestos de trabajo & Disponibilidad producto & 0,000 \\
\hline Crea puestos de trabajo & Reputación de empresa & 0,026 \\
\hline Crea puestos de trabajo & Contribución empresa a obras sociales & 0,032 \\
\hline Crea puestos de trabajo & Imagen de Marca & 0,025 \\
\hline Crea puestos de trabajo & $\begin{array}{l}\text { Necesidad de disponer de información sobre grado RSC } \\
\text { para comprar }\end{array}$ & 0,015 \\
\hline Mejora constantemente calidad & Reputación de empresa & 0,016 \\
\hline Mejora constantemente calidad & Promoción de venta de productos & 0,045 \\
\hline Mejora constantemente calidad & Contribución empresa a obras sociales & 0,035 \\
\hline $\begin{array}{l}\text { Tratamiento similar a todos los } \\
\text { clientes }\end{array}$ & Percepción consumidores sobre producto & 0,023 \\
\hline $\begin{array}{l}\text { Publica informe medioambiental } \\
\text { anual }\end{array}$ & Percepción consumidores sobre producto & 0,012 \\
\hline $\begin{array}{l}\text { Publica informe medioambiental } \\
\text { anual }\end{array}$ & $\begin{array}{l}\text { Necesidad de disponer de información sobre grado RSC } \\
\text { para comprar }\end{array}$ & 0,000 \\
\hline $\begin{array}{l}\text { Contribuye para actividades socio } \\
\text { culturales }\end{array}$ & Contribución empresa a obras sociales & 0,022 \\
\hline $\begin{array}{l}\text { Contribuye para actividades socio } \\
\text { culturales }\end{array}$ & Calidad servicios & 0,015 \\
\hline Tiene precios bajos & Precio producto & 0,019 \\
\hline Obtiene los mayores beneficios & Contribución empresa a obras sociales & 0,002 \\
\hline
\end{tabular}

Fuente: Las encuestas

Elaborado por: la autora

\section{Conclusiones}


No se encuentra relación estadísticamente significativa entre los factores que influyen en la compra de las empresas de elaboración de alimentos y bebidas y la reducción de despilfarro de recursos, el respeto a los derechos humanos, la tenencia de un código ético de conducta, y el ayudar a empleados que desean formarse por parte de los proveedores de envases.

La tabla a continuación resume el número de correlaciones que cada factor de aplicación de RSC posee con los ítems de decisión de compra considerados:

Tabla 7.

Número de correlaciones

\begin{tabular}{lc}
\hline Actividades de RSC & \# de correl. \\
\hline Crea puestos de trabajo & 5 \\
Contribuye mejora calidad de vida & 3 \\
Tiene código ético de conducta & 3 \\
Ofrece trato justo & 2 \\
Precisa info sobre composición productos & 2 \\
Mejora constantemente calidad & 2 \\
Tratamiento similar a todos los clientes & 2 \\
Contribuye para actividades socio culturales & 2 \\
& 1 \\
Invierte en ID en pro del medio ambiente & 1 \\
Ayuda desarrollo país & 1 \\
Rechaza actuar países violan DH & 1 \\
Cumple todas las leyes & 1 \\
Ayuda empleados desean formarse & 1 \\
Publica informe medioambiental anual & 1 \\
Tiene precios bajos & 1 \\
Obtiene los mayores beneficios & \\
\hline \multicolumn{2}{c}{ Fuente: Las encuestas }
\end{tabular}

Se encuentra, por tanto, que la creación de puestos de trabajo, la contribución a la mejora de la calidad de vida, y la tenencia de código ético de conducta por parte del proveedor de envases están relacionadas a una mayor cantidad de criterios de compra.

Finalmente, se aplica correlación chi cuadrado considerando como variables el promedio obtenido, en cuanto, a aplicación de actividades de Responsabilidad Social corporativa por parte del proveedor de envases y el promedio obtenido en los criterios de compra.

Tabla 8.

Pruebas de Chi cuadrado

\begin{tabular}{cccc}
\hline & Valor & gl & Sig. asintótica (2 caras) \\
\hline Chi-cuadrado de Pearson & $383,730^{\text {a }}$ & 325 &, 018 \\
Razón de verosimilitud & 205,323 & 325 & 1,000 \\
Asociación lineal por lineal & 20,611 & 1 &, 000 \\
\hline
\end{tabular}


$\mathrm{N}$ de casos válidos 258

a. 364 casillas $(100,0 \%)$ han esperado un recuento menor que 5 .

Fuente: Las encuestas

Elaborado por: la autora

Por ende, se valida empíricamente que existe una relación entre las actividades de RSC que realizan los proveedores de envases y el puntaje asignados a los criterios de compra considerados.

\section{Recomendaciones}

Al encontrar una relación entre las actividades de RSC y los criterios de compra en el contexto del estudio, se valida que las empresas proveedoras de envases deberían desplegar mayores esfuerzos en aquellas actividades de RSC que guardan correlación con mayor número de criterios de compra a manera de ser más eficiente en el desarrollo de su gestión de RSC.

Es por este motivo que estas empresas deberían desplegar estrategias orientadas a realizar y difundir sus actividades de RSC, sobre todo en cuanto a: la creación de puestos de trabajo, la contribución a la mejora en la calidad de vida, la tenencia de un código ético de conducta, trato justo, información precisa sobre la composición de productos, mejora constante de calidad, tratamiento similar a todos los clientes y contribución a actividades socio culturales.

De igual manera, es necesario destacar que el estudio considera la percepción que los clientes tienen sobre las actividades de RSC que conocen que sus proveedores realizan, por lo que no se considera aquellas actividades de RSC que los proveedores pudieran estar realizando pero no han sido informadas a sus clientes. Por este motivo, futuros estudios deben ahondar en los criterios evaluados y sobre todo en analizar la diferencia entre las actividades de RSC que gestiona la empresa y aquellas actividades que conocen los clientes.

Sin embargo, dado que se ha demostrado la relación entre las actividades de RSC y la decisión de compra, las compañías proveedoras de envases deberían esforzarse no solo en realizar estas actividades, sino también, en comunicar y difundir su gestión y resultados con sus clientes y demás stakeholders para amplificar sus beneficios.

\section{Bibliografía}

Aguilar -Platas, A. y E. Raufflet 2010. Desviación positiva y responsabilidad social empresarial (RSE). En: Innovar. Revista de Ciencias Administrativas y Sociales, no. 20 (37), 21 -32. Altés, C.

Alvarado, A. (2007). Marketing y Responsabilidad Social Empresarial: un mapa de navegación. Teoría y Praxis, 4, 113-148.

Alvarado, A.; Walesska, M. (2008). Dimensionalidad de la responsabilidad social empresarial percibida y sus efectos sobre la imagen y la reputación: una aproximación desde el modelo de Carroll.Estudios Gerenciales, Vol 24. No 108. Julio-Septiembre. Pp 37-59. 
Bhattacharya, C. y Sen, S. (2004). Doing better at doing good: When, why and how consumers respond to corporate social initiatives. California Management Review, 47(1), 9-24.

Bigné, E., Andreu, L., Chumpitaz, R., \& Swaen, V. (2006). La influencia de la responsabilidad social corporativa en el comportamiento de compra de estudiantes universitarios. Esic Market, 6(597), 163-189.

Bigne, E. y Curras,R. (2008). ¿Influye la imagen de responsabilidad social en la intención de compra? El papel de la identificación del consumidor con la empresa. Universia Business Review. Tercer Trimestre 2008. Pp 10-23.

Bigné, E., Chumpitaz R., Andreu, L. y Swaen, V. (2005). "Percepción de la responsabilidad social corporativa: un análisis cross-cultural". Universia Business Review, Primer Trimestre, p. 14-27.

Bravo, R., Fraj, A. E. y Matute, J. (2006). La estrategia de "ecologismo de empresas" en el sector de bienes de consumo final. Universia Business Review, 9, 58-73.

Brown, T. y Dacin, P. (1997). "The company and the product: corporate associations and consumer product responses". Journal of Marketing, Vol. 61 (January), p. 68-84.

Burke, L.; Logsdon, J. (1996). "How Corporate Social Responsibility Pays Off'. Long Range Planning. 29(4), pp.495-502.

Carrigan, M. y Attalla, A. (2001). "The myth of the ethical consumer: do ethics matter in purchase behaviour?”. Journal of Consumer Marketing, Vol. 18, núm. 7, p. 560-577.

Carroll, A. B. (1999). Corporate social responsibility: evolution of a definitional construct. Business \& Society, 38(3), 268-295.

Cervera, A. (2007). Responsabilidad social de la empresa. Aportaciones desde la disciplina del marketing. En P. Giménez, C. de la Calle, y L. Climent (Eds.). Persona y sociedad. Las dos caras del compromise. pp. 131-139.

Correa, M., S, Flynn y A, Amit. (2004). Responsabilidad Social Empresarial en América Latina: una visión empresarial. Santiago de Chile. CEPAL. División de Desarrollo Sostenible y Asentamientos Humanos.

Creyer E., y Ross W. (1997), "The Influence of Firm Behavior on Purchase Intention: Do Consumers Really Care About Business Ethics?" Journal of Consumer Marketing, 14.

Currás, R. (2007). Comunicación de la responsabilidad social corporativa: imagen e identificación con la empresa como antecedentes del comportamiento del consumidor. Tesis doctoral. Universitat de València, España. 
Ellen, P. S., Webb, D. J. y Mohr, L. A. (2006). Building corporate associations: consumer attributions for corporate socially responsible programs. Journal of the Academy of Marketing Science, 34(2),147-157.

Fernández, D. y Merino, A. (2005). “¿Existe disponibilidad a pagar por responsabilidad social corporativa? Percepción de los consumidores". Universia Business Review, Tercer Trimestre, p. 2-17.

Fundación ADECCO.(2014) Las empresas del sector Alimentación y Bebidas, las más responsables según los ciudadanos españoles. Madrid: Fundación Adecco. 6 p. En: http://www.fundacionadecco. es/_data/SalaPrensa/SalaPrensa/Pdf/583.pdf

García de los Salmones, M. d. M. (2002). La imagen de empresa como factor determinante en la elección de operador. Universidad de Cantabria, España.

Garriga, E. y Melé, D. (2004). Corporate social responsibility theories: mapping the territory. Journal of Business Ethics, 53(1-2), 51-71.

Guardela, L. y Barrios, I. (2006) Colombia: ¿En la vía del desarrollo sostenible? En: Revista de Derecho. no. 26, p. 110- 136.

Hadjikhani, A., Lee, J., \& Park, S. (2016). Corporate social responsibility as a marketing strategy in foreign markets. international Marketing Review, 33(4), 530-554.

IADB (2004). Corporate Social Responsibility in Latin America, unpublished report. Washington, D.C.: InterAmerican Development Bank.

IFC (2002). Investing in a Sustainable Private Sector. Annual Report. Worl Bank Group. http://www.ifc.org/wps/wcm/connect/bc8ce20046d82782a62bae5198539f7e/AR2002_En glish_vol1.pdf?MOD=AJPERES.

Iglesias,O. Ind,N. y Alfaro,M. (2013). The organic view of the Brand: a brand value co-creation model. Journal of brand Management. Vol 20. No 8. Pp. 670-688.

Instituto Colombiano de Normatización y Certificación.(2012) Norma ISO sobre responsabilidad social para aprobación. Bogotá D.C: ICONTEC. Disponible en http://www.icontec.org. co/

Jüttner, U. Wehrli, H. (1994) "Competitive Advantage: Merging Marketing and the Competence based Perspective", Journal of Business \& Industrial Marketing, Vol. 9 Iss: 4, pp.42 - 53.

Keller, K. (1993). “Conceptualizing, Measuring and Managing Customer Based Brand Equity”.Journal of Marketing, January, Vol. 57, p. 1-22.

Kolk,A. Van Tulder,R. (2010). International business, corporate social responsibility and sustainable development. Int.Bus.Rev. Rev19(2). Pp. 119-125 
Kraus,P. y Britzelmaier,B. (2012). A literatura review on corporate social responsibility:definitions, theories and recent empirical research. International Journal of Management Cases. Vol 14. No 4. Pp. 282-296

Lizcano, J. L. y Nieto, P. (2006). La semántica de la responsabilidad social corporativa. Madrid, España:AECA.

López, A. , Contreras, R. , Molina, R.(2011) La Responsabilidad social empresarial como estrategia de competitividad en el sector alimentario. En: Cuadernos de Administración. Julio-Diciembre, vol.24 no.43, p. 261-283.

Marquina, P. y Morales, C. (2012). The influence of CSR on purchasing behaviour in Peru and Spain. International Marketing Review, 29(3), 299-312.

Macías,M. (2006). Elaboración de un plan maestro de producción y de gestión tecnológica en la empresa CRIDESA. Universidad de Guayaquil. Facultad de Ingeniería Industrial.Guayaquil, Ecuador.

McWilliams, A., Siegel, D. S. y Wright, P. M. (2006). Corporate social responsibility: strategic implications (editorial). Journal of Management Studies, 43(1), 1-18.

Meyvis,T. Goldsmith,K. \& Dhar,R. (2012). The importance of the context in brand extensions: how pictures and comparisons shift consumers focus from fit to quality. Journal of marketing research. Vol XLIX. No2. Pp. 206-217.

Méndez, M. T. (2005). Ética y responsabilidad social corporativa. En: Ética y Economía, Junio 2005, No 823. Madrid. ICE.

Ministerio de Ambiente, Vivienda y Desarrollo Territorial de Colombia.(2007) Iniciativa Latinoamericana y Caribeña para el Desarrollo Sostenible: indicadores de seguimiento. Colombia. Bogotá: Ministerio de Medio Ambiente, Vivienda y Desarrollo Territorial (MAVDT), Departamento Administrativo Nacional de Estadísticas (DANE), Programa de las Naciones Unidas para el Medio Ambiente, 102 p.

Park,B.; Chidlow,A. \& Choi,J. (2014). Corporate social responsibility:stakeholders influence on MNE's activities. International Business Review. Vol.23.No5. pp.966-980.

Parlamento Europeo. A7-0017/2013 (28, Enero, 2013). Informe sobre responsabilidad social de las empresas: comportamiento responsable y transparente de las empresas y crecimiento sostenible. 2013. $46 \mathrm{p}$.

Polonsky, M. J., \& Jevons, C. (2006). Understanding issue complexity when building a socially responsible brand. European business review, 18(5), 340-349.

Rindell,A. (2013). Time in corporate images: introducing image heritage and image-in-use. Qualitative Market Research, An International Journal. Vol 16. No 2. Pp. 197-213 
Steffen,W. Broadgate,W. Deutsch,L. Gaffney,O. Ludwig,C. (2015). The trajectory of the anthropocene:the great acceleration. Anthr. Rev.2 (1). Pp.81-98

Superintendencia de Companias del Ecuador (2016) Portal de información de sector societario. Recuperado de: http://appscvs.supercias.gob.ec/portalInformacion/sector_societario.zul

Van Herpen, E., Pennings, J. M. y Meulenberg, M. (2003). Consumers' evaluations of socially responsible activities in retailing.

Vargas, J. (2005). Responsabilidad Social Empresarial (RSE) desde la perspectiva de los consumidores. Documento de proyecto. CEPAL. Santiago de Chile. Ed. Naciones Unidas. http://www.utopia.pcn.net/pobreza. html. Última visita: 20 febrero 2014.

Villafañe, J. (1996). Principios de teoría general de la imagen, Madrid, España: Pirámide.

Webb, D. J. y Mohr, L. A. (1998). A typology of consumer responses to causerelated marketing: from skeptics to socially concerned. Journal of Public Policy \& Marketing, 17(2),pp. 226 238.

Wright, P.; Ferris, S. (1997). "Agency conflict and corporate strategy: The effect of disvestment on corporate value", Strategic Management Journal, 18(1), pp 77-83 